\title{
Recurrent Spontaneous Pneumothorax during the Recovery Phase of ARDS Due to $\mathrm{H}_{1} \mathrm{~N}_{1}$ Infection
}

\author{
Canan Bor ${ }^{1}$, Kubilay Demirağ ${ }^{1}$, Mehmet Uyarr ${ }^{1}$, Ilkin Çankayalı ${ }^{1}$, Ali Reşat Moral ${ }^{2}$ \\ ${ }^{1}$ Department of Anesthesiology and Reanimation, Faculty of Medicine, Ege University, Izmir, Turkey \\ ${ }^{2}$ Clinic of Anesthesiology and Reanimation, Special izmir City Hospital, Izmir, Turkey
}

\begin{abstract}
The pregnant patients are prone to influenza $A\left(H_{1} N_{1}\right)$ virus infection, which may rapidly progress to lower respiratory tract infection and subsequent respiratory failure and acute respiratory distress syndrome (ARDS). Pneumothorax might develop in ARDS under mechanical ventilation. But post-ARDS pneumothorax in spontaneously breathing patient has not been reported in the literature. We report a 31 -year old pregnant woman infected with influenza $A\left(\mathrm{H}_{1} \mathrm{~N}_{1}\right)$ virus and progressed to ARDS. Mechanical ventilation with high PEEP improved patient's gas exchange parameters within 3 weeks. However spontaneous pneumothorax was developed one week after she weaned off the ventilator. After successful drainage therapy, the patient was discharged. However she re-admitted to the hospital because of a recurrent pneumothorax one week later. She was discharged in good health after being treated with negative continuous pleural aspiration for 10 days. Influenza might cause severe pulmonary infection and death. In addition to diffuse alveolar damage, sub-pleural and intrapulmonary air cysts might occur in influenza-related ARDS and may lead to spontaneous pneumothorax. This complication should always be considered during the recovery period of ARDS and a long-term close follow-up is necessary.
\end{abstract}

Key Words: $\mathrm{H}_{1} \mathrm{~N}_{1}$ virus, spontaneous pneumothorax, ARDS

Received: 29.05.2012

Accepted: 10.09 .2012

\section{Introduction}

The novel influenza $A\left(\mathrm{H}_{1} \mathrm{~N}_{1}\right)$ pandemic has affected Turkey during 2009. Pregnant women might be at increased risk for complications due to pandemic $\mathrm{H}_{1} \mathrm{~N}_{1}$ viral infection (1). $\mathrm{Pa}$ tients with influenza $A\left(\mathrm{H}_{1} \mathrm{~N}_{1}\right)$ infection may develop lower respiratory tract infection rapidly resulting in respiratory failure and acute respiratory distress syndrome (ARDS) (2). The incidence of pneumothorax in ARDS is high and is the most commonly encountered manifestation of lung damage induced by barotrauma. Spontaneous pneumothorax rate after ARDS is unknown. To our knowledge spontaneous pneumothorax due to swine-flu pneumonia was not reported. We present here an unusual case, who was infected with influenza A during pregnancy and developed ARDS and recurrent pneumothorax secondary to pneumonia.

\section{Case Report}

A 31-year-old 27-28 weeks pregnant woman was admitted to a local hospital because of high fever, cough and myalgia. She had been healthy until five days earlier. She was diagnosed as bilateral pneumonia (Figure 1) and the patient was transferred to intensive care unit. Two days after admission, she was sent to a university hospital because of refractory hypoxemia.

After admission to the intensive care unit (ICU) of University Hospital, she had an urgent caesarean section (C-section) under general anesthesia and a $1070 \mathrm{~g}$ premature baby girl was transferred to the newborn unit. After the operation, she was brought to ICU sedated and paralyzed. Initially she was ventilated by pressure-controlled ventilation, with $\mathrm{FiO}_{2} 0.90$, respiration rate 20 breath/min, PEEP $14 \mathrm{~cm} \mathrm{H}_{2} \mathrm{O}, \mathrm{P}_{\text {peak }} 35 \mathrm{~cm}$ $\mathrm{H}_{2} \mathrm{O}$. Chest CT showed bilateral consolidation and diffuse infiltrates (Figure 2). She was stable hemodynamically. Nasal and throat swabs for influenza were taken and $\mathrm{H}_{1} \mathrm{~N}_{1}$ infection was confirmed with real time reverse transcriptase-polymerase chain reaction (RT-PCR). Oseltamivir (a neuraminidase inhibitor) and antibiotic therapy were started. On the $4^{\text {th }}$ day on mechanical ventilation, chest X-ray showed bilateral pleural effusion. $800 \mathrm{ml}$ of pleural effusion was drained from left side by small-bore catheter (Pleuracan ${ }^{\circledR}$ ) and removed 4 days later. Despite high PEEP, recruitment maneuvers and prone positioning, $\mathrm{FiO}_{2}$ remained over 0.8 and $\mathrm{SpO}_{2}$ between $90-94 \%$. Tracheotomy was performed 9 days after admission. She was responded well to $2^{\text {nd }}$ and $3^{\text {rd }}$ attempts of prone positioning, and $\mathrm{FiO}_{2}$ was reduced to 0.6. After Acinetobacter sp. growth in mini-BAL specimen, she was considered as ventilatoryassociated pneumonia and tigecycline was started. Weaning was started on pressure support ventilation at $22^{\text {nd }}$ day of ICU stay. She was able to tolerate spontaneous ventilation, and successfully decannulated 26 days after admission. Two days after decannulation, agitation and hallucinations were observed and she was consulted by psychiatry and diagnosed as delirium. While she was stable and ready to discharge from ICU, suddenly dyspnea started on $37^{\text {th }}$ of admission. She was 
mildly tachypneic and a hyperresonant on percussion over the right hemithorax was detected. Oxygen saturation was $90 \%$ on room air. Chest X-ray revealed a collapsed lung and spontaneous pneumothorax on right side. Pneumothorax was treated by tube thoracostomy and resolved completely in five days. During this period she did not need mechanical ventilation. She was discharged from ICU 45 days after admission and stayed two more days in the ward.

Seven days after hospital discharge, she readmitted to our hospital's emergency department because of shortness of breath. She was found to be mildly tachypnoeic. Chest $X$-ray and CT scan were found normal. She was monitored and treated with oxygen $5 \mathrm{~L} / \mathrm{min}$ via face mask. She was increasingly dyspneic at $7^{\text {th }}$ hour after admission and a second chest X-ray revealed right pneumothorax. Pneumothorax was treated by chest tube and after that she was admitted to ICU (Figure 3). She was stable on spontaneous ventilation via face mask. Control chest X-Ray showed that right lung was not to-

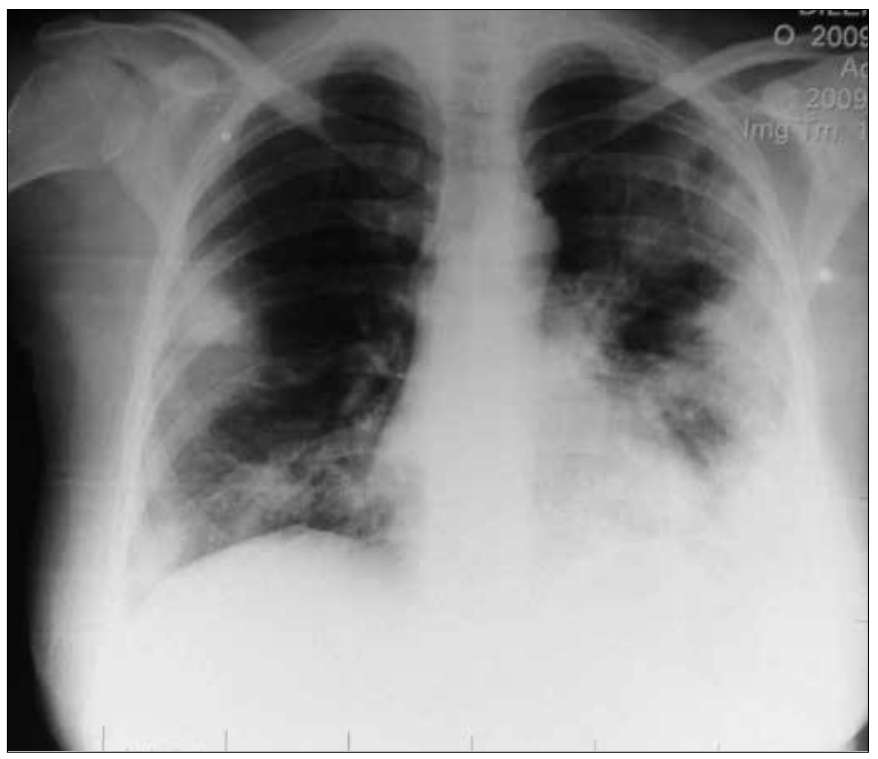

Figure 1. Chest X-Ray showing bilateral pneumonia

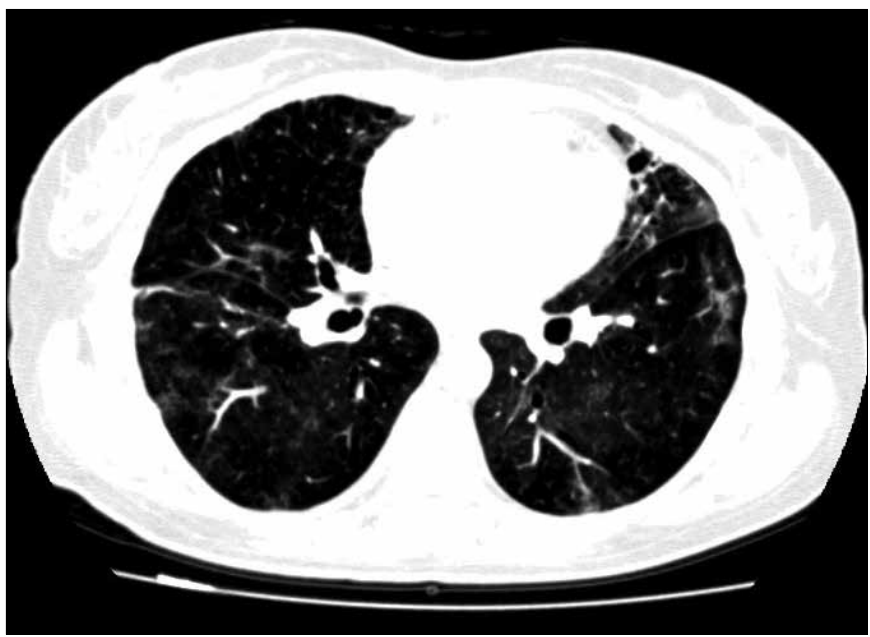

Figure 2. Chest CT indicating bilateral consolidation and diffuse infiltrates tally expanded. Right pneumothorax and pleural effusion and bilateral fibrosis were detected on high resolution computed tomography (HRCT) scan. Closed chest tube drainage was attached and $15-20 \mathrm{mmHg}$ of negative pressure was maintained. While the right lung was not re-expanded totally even with continuous suction for 11 days, she was transferred to thoracic surgery ward. She was discussed for pleural autologous blood patch but was not performed and lung was expanded 20 days later. She was discharged from hospital in healthy condition at the $24^{\text {th }}$ day of her $2^{\text {nd }}$ admission. She is still under long-term follow-up.

\section{Discussion}

The mechanical, immunological, and hormonal changes of pregnancy might contribute to increased risk of infection for both mother and baby during pandemic or seasonal influenza. During the previous two influenza pandemics, maternal mortality was significantly higher (3). Pregnant women were 4 times more likely to be hospitalized than the general population when infected with this virus (1). It was reported that total number of deaths due to $\mathrm{H}_{1} \mathrm{~N}_{1}$ pandemic in Turkey was 627 between April-January 2009, and 40 of them were pregnant (4). Our case was pregnant and infected by $\mathrm{H}_{1} \mathrm{~N}_{1}$ virus. Upper respiratory tract sampling using a combination of nasal or nasopharyngeal and throat swab is advised by the World Health Organization (WHO2009 $\mathrm{H}_{1} \mathrm{~N}_{1}$ ) for diagnosis and confirmation $\mathrm{H}_{1} \mathrm{~N}_{1}$ infection with real time reverse transcriptase-polymerase chain reaction (RT-PCR). This test was performed in our case after admission to ICU. Influenza might cause severe illness and death in pregnant and postpartum women; regardless of the results of rapid antigen testing, prompt evaluation and antiviral treatment of influenza-like illness should be considered in such women (5). Early treatment with antiviral medications is optimal to reduce illness in seasonal influenza infection (6). We treated our patient with oseltamivir after admission to the intensive care unit. The US Centers for Disease Control and Prevention (CDC) reported that deaths in pregnant women were all related with pneumonia and subsequent acute respiratory

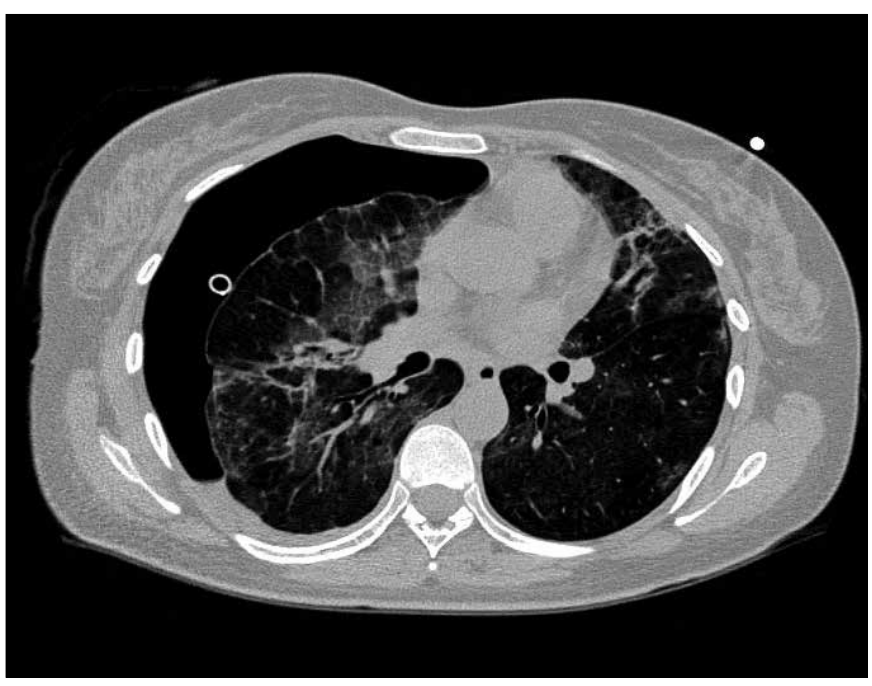

Figure 3. Chest CT showing pneumothorax 
distress syndrome requiring mechanical ventilation (1). Likewise, our case developed pneumonia and required mechanical ventilation. $\mathrm{H}_{1} \mathrm{~N}_{1}$ infection-related viral pneumonia was associated with focal to extensive diffuse alveolar damage, marked hyaline membrane formation, pulmonary edema and acute pulmonary hemorrhage (7). ARDS was seen more frequently in pregnancy compared to the 1.5 cases per 100.000 per year for the general population. Some obstetric causes leading to ARDS requiring prolonged ventilator support are preeclampsia, amnionitis-endometritis, obstetric hemorrhage and tocolytic therapy (8). In our case, $\mathrm{H}_{1} \mathrm{~N}_{1}$ pneumonia progressed to ARDS and even pregnancy was terminated at $28^{\text {th }}$ weeks of gestation by emergency C-section.

The ARDS lung is stiff from both alveolar and interstitial edema and physiologically small. During mechanical ventilation, PEEP inflates and recruits some of the collapsed regions, but also overinflates the normal regions. The lung regions subjected to high-pressure overinflation may develop alveolar rupture resulting air tracking (9). Our case was treated for ARDS and required high PEEP, recruitment maneuvers and prone positioning, to keep oxygenation level optimal during mechanical ventilation therapy. Despite an uneventful clinical course during invasive mechanical ventilation, she developed pneumothorax while breathing spontaneously room air after decannullation. Spontaneous pneumothorax is a pathologic condition of extrapulmonary air within the chest accompanied by lung collapse without trauma to the lung or chest wall. It is encountered more frequently in males than in females. Rupture of small bullae has been suggested as one cause for primary spontaneous pneumothorax, while secondary spontaneous pneumothorax develop in the setting of pre-existing lung disease such as chronic obstructive pulmonary disease (COPD), asthma, tuberculosis, pneumonia, cystic fibrosis, lung malignancies, and certain types of interstitial lung disease (10). Sub-pleural and intrapulmonary air cysts occur in ARDS patients. Gattinoni et al. (11) found that late ARDS had an increased incidence of pneumothoraces when compared to early ARDS patients ( $87 \%$ vs $30 \%$ ), an increased number of bullae, and decreased lung compliance. In this case, due to ARDS, bullae might have occurred and post-ARDS spontaneous pneumothorax developed. But we were unable to detect this formation by $x$-ray and chest $\mathrm{CT}$. Spontaneous recurrent pneumothorax is an unusual condition and its exact incidence is unknown. Among patients with pneumothorax, 30 to $38 \%$ of them have recurrence (12). Although primary spontaneous pneumothorax usually resolves in 2 to 4 days of drainage, pneumothoraces in patients with underlying lung disease often last substantially longer (13). In our case, post-ARDS recurrent pneumothorax needed drainage for three weeks.

According to our knowledge, this is the first report of recurrent pneumothorax due to $\mathrm{H}_{1} \mathrm{~N}_{1}$ viral pneumonia-related ARDS. The second pneumothorax developed while the patient was at home. The first event also might have been occurred at home if her discharge was not postponed because of delirium. Recurrent spontaneous pneumothorax during the recovery phase of ARDS due to $\mathrm{H}_{1} \mathrm{~N}_{1}$ infection may occur, therefore usual discharge criteria should be questioned in such patients.
Diffuse alveolar damage, sub-pleural and intrapulmonary air cysts might occur in influenza-related ARDS and may lead to spontaneous pneumothorax. Recovery period of ARDS and a long-term close follow-up is necessary.

Informed Consent: Written informed consent was obtained from patients who participated in this study.

Peer-review: Externally peer-reviewed.

Author contributions: Concept - C.B.,M.U.,K.D.,I.Ç.; Design C.B.,M.U.; Supervision - M.U.; Resource - C.B., K.D., M.U., I.Ç., A.R.M.; Materials - C.B., K.D., M.U., I.Ç., A.R.M.; Data Collection\&/or Processing - C.B., K.D., M.U., I.Ç., A.R.M.; Analysis\&/or Interpretation - M.U., C.B.; Literature Search - C.B., K.D.,M.U.; Writing - C.B., K.D., M.U.; Critical Reviews - C.B., M.U., K.D., I.C.

Conflict of Interest: No conflict of interest was declared by the authors.

Financial Disclosure: No financial disclosure was declared by the authors.

\section{References}

1. Jamieson DJ, Honein MA, Rasmussen SA, Williams JL, Swerdlow DL, Biggerstaff MS, et al. H1N1 2009 influenza virus infection during pregnancy in the USA. Lancet 2009;374:451-8. [CrossRef]

2. Rello J, Rodríguez A, Ibañez P, Socias L, Cebrian J, Marques A, et al. Intensive care adult patients with severe respiratory failure caused by Influenza A (H1N1)in Spain. Crit Care 2009;13:R148. [CrossRef]

3. Goognight $\mathrm{WH}$, Soper DE. Pneumonia in pregnancy. Crit Care Med 2005;33:390-7. [CrossRef]

4. Turkish Ministry of Health (unpublished data) www.rsmh.gov.trwww.grip.gov.tr (19.1.2010)

5. Louie JK, Acosta M, Jamieson DJ, Honein MA;California Pandemic (H1N1) Working Group. Severe 2009 H1N1 influenza in pregnant and postpartum women in California. N Engl J Med 2010;362:27-35. [CrossRef]

6. Harper SA, Bradley JS, Englund JA, File TM, Gravenstein S, Hayden FG, et al. Seasonal influenza in adults and children--diagnosis, treatment, chemoprophylaxis, and institutional outbreak management:clinical practice guidelines of the Infectious Diseases Society of America. Clin Infect Dis 2009;48:1003-32. [CrossRef]

7. Gill JR, Sheng ZM, Ely SF, Guinee DG, Beasley MB, Suh J, et al. Pulmonary pathologic findings of fatal 2009 pandemic influenza A/H1N1 viral infections. Arch Pathol Lab Med 2010;134:235-43.

8. Catanzarite VA, Willms D. Adult respiratory distress syndrome in pregnancy:report of three cases and review of the literature. Obstet Gynecol Surv 1997;52:381-92. [CrossRef]

9. Jain P, Goswami K. Recurrent pneumothorax during pregnancy:a case report. J Med Case Rep. 2009;3:81.[CrossRef]

10. Wennergren $M$, Jorgensen $C$, Bugge $M$, Lepore $V$, Gatzinsky P. Pneumothorax in pregnancy. Acta Obstet Gynecol Scand 1990;69:441-2. [CrossRef]

11. Gattinoni L, Bombino M, Pelosi P. Lung structure and function in different stages of severe adult respiratory distress syndrome. JAMA 1994;271:1772-9. [CrossRef]

12. Bryant $\mathrm{L}$, Morgan $\mathrm{S}$. Chest wall, pleura lung, and mediastinum. In:Schwartz SI, Shires GT, Spencer FC, Storer EH, editors. Principles of surgery, 4th ed. New York, Toronto:McGrawHill;1984:675-7.

13. Chee CB, Abisheganaden J, Yeo JK, Lee P, Huan PY, Poh SC, et al. Persistent air-leak in spontaneous pneumothorax--clinical course and outcome. Respir Med 1998;92:757-61. [CrossRef] 